\title{
Assessing drug efficacy against Plasmodium falciparum liver stages in vivo
}

Erika L. Flannery, ${ }^{1}$ Lander Foquet, ${ }^{1}$ Vorada Chuenchob, ${ }^{1}$ Matthew Fishbaugher, ${ }^{1}$ Zachary Billman, ${ }^{2}$ Mary Jane Navarro, ${ }^{1}$ William Betz, ${ }^{1}$ Tayla M. Olsen, ${ }^{2}$ Joshua Lee, ${ }^{2}$ Nelly Camargo, ${ }^{1}$ Thao Nguyen, ${ }^{1}$ Carola Schafer, ${ }^{1}$ Brandon K. Sack,, Elizabeth M. Wilson, ${ }^{3}$ Jessica Saunders, ${ }^{4}$ John Bial, ${ }^{3}$ Brice Campo, ${ }^{5}$ Susan A. Charman, ${ }^{4}$ Sean C. Murphy, ${ }^{2}$ Margaret A. Phillips, ${ }^{6}$ Stefan H.I. Kappe, ${ }^{1,7}$ and Sebastian A. Mikolajczak'

${ }^{1}$ Center for Infectious Disease Research, Seattle, Washington, USA. ${ }^{2}$ Departments of Laboratory Medicine and Microbiology, University of Washington, Seattle, Washington, USA. ${ }^{3}$ Yecuris Corporation, Portland, Oregon, USA. ${ }^{4}$ Centre for Drug Candidate Optimisation, Monash Institute of Pharmaceutical Sciences, Monash University, Parkville, Victoria, Australia. ${ }^{5}$ Medicines for Malaria Venture, Geneva, Switzerland. ${ }^{6}$ Department of Pharmacology, University of Texas Southwestern Medical Center at Dallas, Dallas Texas, USA. 'Department of Global Health, University of Washington, Seattle Washington, USA.

Malaria eradication necessitates new tools to fight the evolving and complex Plasmodium pathogens. These tools include prophylactic drugs that eliminate Plasmodium liver stages and consequently prevent clinical disease, decrease transmission, and reduce the propensity for resistance development. Currently, the identification of these drugs relies on in vitro $P$. falciparum liver stage assays or in vivo causal prophylaxis assays using rodent malaria parasites; there is no method to directly test in vivo liver stage activity of candidate antimalarials against the human malaria-causing parasite $P$. falciparum. Here, we use a liver-chimeric humanized mouse (FRG huHep) to demonstrate in vivo $P$. falciparum liver stage development and describe the efficacy of clinically used and candidate antimalarials with prophylactic activity. We show that daily administration of atovaquone-proguanil (ATQ-PG; ATQ, $30 \mathrm{mg} / \mathrm{kg}$, and PG, $10 \mathrm{mg} / \mathrm{kg}$ ) protects 5 of 5 mice from liver stage infection, consistent with the use in humans as a causal prophylactic drug. Single-dose primaquine $(60 \mathrm{mg} / \mathrm{kg})$ has similar activity to that observed in humans, demonstrating the activity of this drug (and its active metabolites) in FRG huHep mice. We also show that DSM265, a selective Plasmodial dihydroorotate dehydrogenase inhibitor with causal prophylactic activity in humans, reduces liver stage burden in FRG huHep mice. Finally, we measured liver stage-to-blood stage transition of the parasite, the ultimate readout of prophylactic activity and measurement of infective capacity of parasites in the liver, to show that ATQ-PG reduces blood stage patency to below the limit of quantitation by quantitative PCR (qPCR). The FRG huHep model, thus, provides a platform for preclinical evaluation of drug candidates for liver stage causal prophylactic activity, pharmacokinetic/pharmacodynamics studies, and biological studies to investigate the mechanism of action of liver stage active antimalarials.

Conflict of interest: MAP and SAC hold a patent (US 9,238,653 B2) covering DSM265 and related compounds.

Submitted: January 5, 2017 Accepted: November 21, 2017 Published: December 21, 2017

Reference information: JCI Insight. 2017;2(24): e92587. https://doi.org/10.1172/jci. insight. 92587

\section{Introduction}

Malaria eradication is a prominent global health goal. While pronounced advances have been made in decreasing the incidence of $P$. falciparum in the last 15 years (1), new tools must continue to be developed that appropriately support eradication efforts. These will include, but are not limited to, antimalarials with novel mechanisms of action that eliminate drug-resistant parasites already present in the field, as drug resistance has emerged at varying degrees to all current antimalarials (2, 3). Ideally, these novel antimalarials will also have prophylactic activity, preventing clinical disease and parasite transmission $(4,5)$.

The liver stages of Plasmodium, constituting a severe bottleneck in the life cycle, present a unique opportunity for prophylactic intervention. When a mosquito takes a bloodmeal, only tens to a few hundred sporozoites are transmitted to the host - each sporozoite is potentially able to initiate hepatocyte infection and form a liver 
stage (6). This is substantially smaller than parasite numbers in the blood stage infection, which can exceed billions (7). Eliminating parasites in the liver prevents disease in the individual and eliminates transmission, as parasites never reach the blood stage. Furthermore, it also reduces the propensity for resistance development due to the fewer parasites present in the liver and the acyclic nature of exoerythrocytic schizogony.

Historically, antimalarials have been developed to cure blood stage infection, the symptomatic and potentially lethal stage of the disease, yet many of these drugs do not have activity against the liver stage of the parasite. Therefore, of the many antimalarial candidates currently in late product development, few have potential activity against $P$. falciparum liver stages while also acting through novel targets in the parasite (8). While the rodent malaria models are used for preliminary testing of antimalarial drug candidates in vivo, fundamental differences in rodent and human malaria parasites exist. Most importantly, the rodent parasite $P$. berghei undergoes complete liver stage development within 50 hours of infection, while complete liver stage development of $P$. falciparum occurs between 5.5 and 6.5 days (9). This is an important distinction when drug half-lives and dosing regimens are extrapolated between species. Another important limitation of the rodent model is genetic dissimilarities between model organisms and the human malaria parasites potentially confounding the evaluation of target-specific compounds. For example, analogues of the dihydroorotate dehydrogenase (DHODH) inhibitor DSM265 were 100-fold less potent against $P$. berghei $\mathrm{DHODH}$ compared with $P$. falciparum $\mathrm{DHODH}(10)$.

There is no preclinical in vivo model to test candidates for activity against $P$. falciparum liver stages. We previously developed a human liver-chimeric mouse model that supports complete $P$. falciparum liver stage development with kinetics similar to those in humans (11). In this model, immunocompromised $\left(\mathrm{Rag}^{-/-}, \mathrm{IL} 2 \mathrm{R} \gamma^{\text {null }}\right)$ mice also harbor a fumarylacetoacetate hydrolase deletion $\left(\mathrm{FAH}^{-/-}\right)$, which causes liver disease and murine hepatocyte damage $(12,13)$. This murine hepatocyte death, which can be rescued by the addition of 2-(2-nitro-4-trifluoromethylbenzoyl)-1, 3-cyclohexanedione (NTBC) to the drinking water, allows for repopulation with healthy primary human hepatocytes that functionally support mouse processes and full liver stage development of human malaria $(11,14)$. We herein show that this model (FRG huHep) can be employed to test the liver stage efficacy of existing drugs and potentially novel antimalarial candidates against $P$. falciparum and, thus, is a useful preclinical model for introduction into the antimalarial drug development pipeline.

\section{Results}

Infection by mosquito bite yields robust $P$. falciparum liver stage burden. To mimic the natural route of infection, we compared the effectiveness of FRG huHep infection by mosquito bite versus i.v. injection of sporozoites. First, to ensure the consistency of human hepatocyte repopulation and therefore direct comparisons between mice, we measured levels of human GAPDH expression in FRG huHep livers (Figure 1A). We also compared human albumin levels between control groups used for the experiment (Figure 1B). No significant difference was observed in albumin levels or human GAPDH gene expression in the FRG huHep mice showing equal human hepatocyte repopulation between the mice. $P$. falciparum luciferase-expressing (NF54HT-GFP-luc) sporozoites (15) were introduced either by tail vein injection of $1 \times 10^{6}$ sporozoites or by the bite of 50 infected mosquitoes. Six days after challenge, when liver burden is highest, liver stage $P$. falciparum infection was measured using in vivo bioluminescence imaging (IVIS), as well as liver harvest followed by quantification of parasite 18S rRNA by quantitative reverse transcription PCR (qRT-PCR) (Figure 1C). There was no significant difference in liver burden between the mice infected by i.v. tail vein injection or mosquito bite, and quantification of parasite liver burden by IVIS and qPCR was correlated (Pearson $r^{2}=0.53, P<0.001$ ) (Figure 1D), suggesting either method of inoculation and either method of quantification is suitable for establishment and measurement of $P$. falciparum liver infection in FRG huHep mice.

To establish the humanized mouse as a viable platform for the identification of drugs with causal prophylactic activity, we used an antimalarial formulation currently recommended for the prevention of malaria infection in travelers. FRG huHep mice were infected with $P$. falciparum by the bites of 50 infected mosquitoes. Five mice were treated with $30 \mathrm{mg} / \mathrm{kg}$ atovaquone (ATQ) and $10 \mathrm{mg} / \mathrm{kg}$ proguanil (PG) combination therapy 24 hours prior to infection and for 6 subsequent days after infection. Six days after infection, the average IVIS signal was $6.8 \times 10^{4}$ photons $/ \mathrm{sec} / \mathrm{cm}^{2}$ in the control group and below background levels in all 5 mice in the ATQ-PG-treated group (Figure 1, E and F), demonstrating the causal prophylactic activity of this drug combination in the FRG huHep platform. 


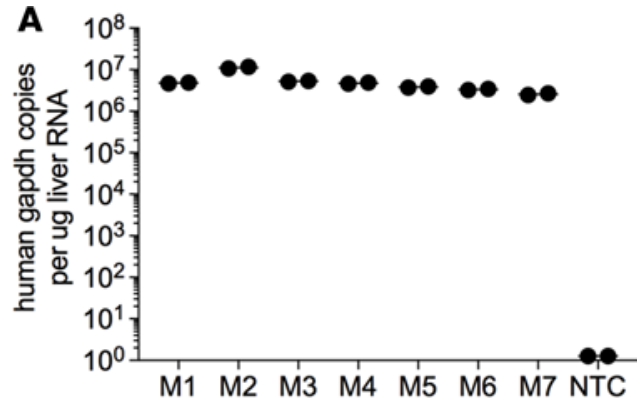

C
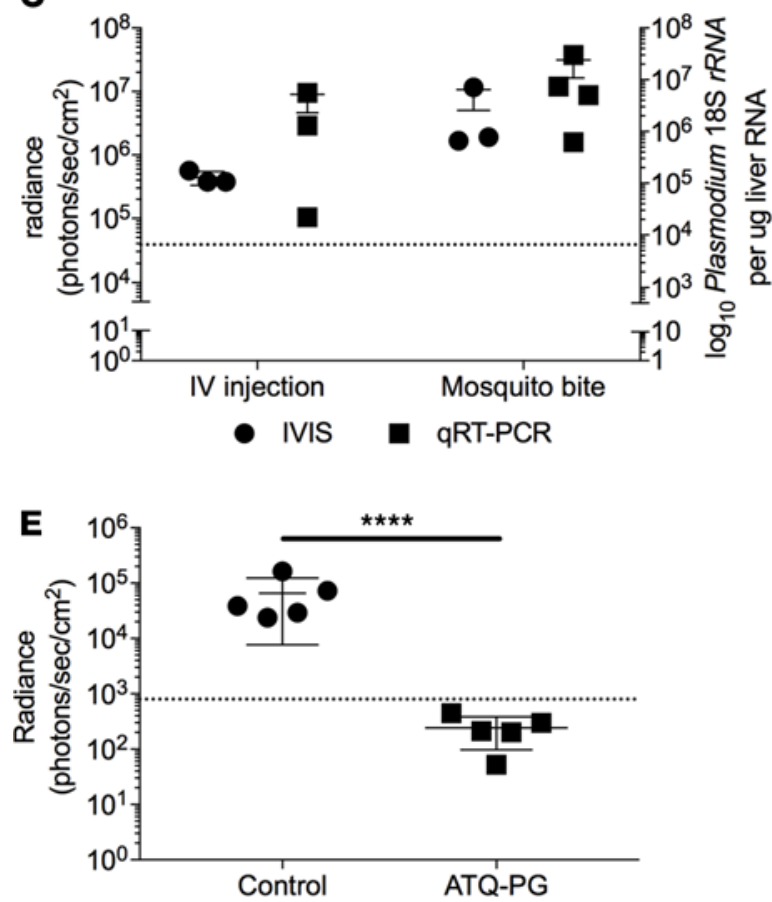

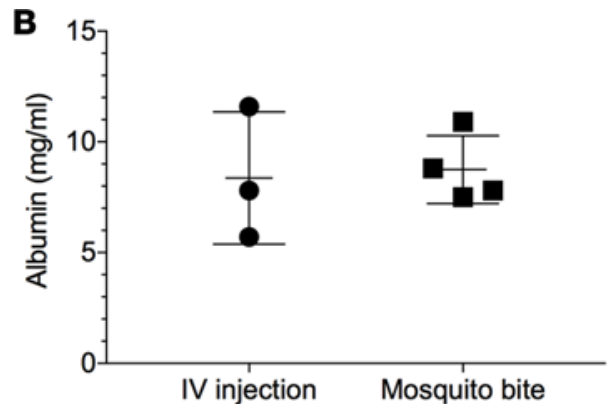

D

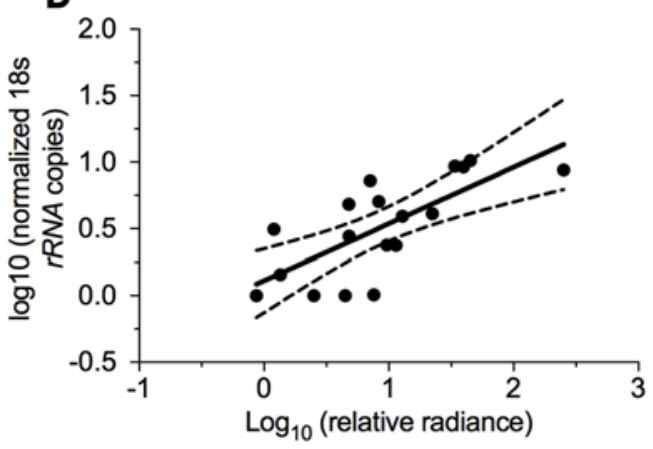

$\mathbf{F}$

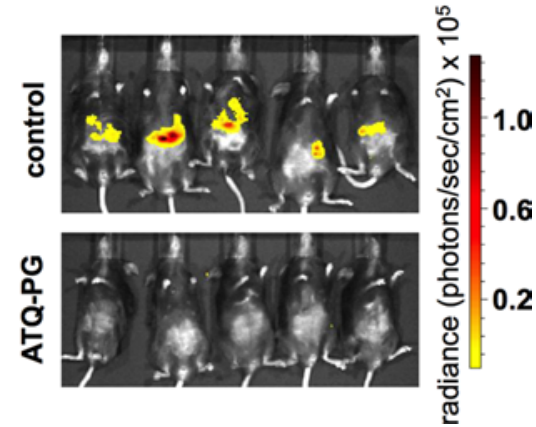

Figure 1. $\boldsymbol{P}$. falciparum liver infection in FRG huHep mice is established by mosquito bite and prevented with atovaquone-proguanil. (A) Human $G A P D H$ transcript levels measured by qPCR in duplicate in 7 different FRG huHep mice. M, mouse; NTC, no template control. (B) Human albumin levels measured prior to FRG huHep infection. (C) P. falciparum NF54HT-GFP-luc liver stage burden 6 days after infection measured by in vivo immunoflourescence (IVIS) or 185 rRNA qPCR. Mice were infected with either $1 \times 10^{6}$ freshly dissected sporozoites by i.v. injection or the direct feeding of 50 infected mosquitoes. Error bars for qPCR measurements represent mean \pm SEM. (D) Correlation of liver burden estimates using qPCR and IVIS. Pearson correlation $r^{2}=0.53, P<$ 0.001. Dashed lines denote upper and lower $95 \% \mathrm{CI}$. (E) Radiance levels 6 days after infection after treatment with atovaquone-proguanil (ATQ-PG) from day -1 to day 6 . Mice were infected by the bite of 50 infectious mosquitoes. Unpaired 2-tailed Student's $t$ test, ${ }^{* * * *} P<0.0001$. (F) IVIS images of luminescence in livers of live FRG huHep mice from (E). Each symbol represents 1 mouse (B-E). Dashed line represents background radiance (C and $\mathbf{E})$.

P. falciparum liver infection is reduced by the DHODH inhibitor, DSM265. DSM265 is a new antimalarial molecule that selectively targets Plasmodial DHODH and exhibits potent blood stage activity against $P$. falciparum both in vitro and in vivo, as well as good liver stage activity in vitro (16). Therefore, we were interested in assessing the activity of DSM265 in the FRG huHep model and in understanding its effect on P. falciparum liver stage development in vivo. First, to understand the in vivo disposition of DSM265 in FRG huHep mice, we treated mice orally with $20 \mathrm{mg} / \mathrm{kg}$ DSM265 (formulated as a spray-dried dispersion at $25 \%$ [250 mg/g] drug load in hydroxymethylcellulose acetate succinate [HPMCAS-MG]) and measured blood concentrations over time (Figure 2A). Mice were given 3 doses of $20 \mathrm{mg} / \mathrm{kg}$ DSM265, 24 hours apart. The 24- and 48-hour blood samples were collected prior to the daily administration of DSM265 and therefore represent minimum concentrations. After the first dose, the DSM265 concentration at 6 hours $(12.9 \mu \mathrm{g} / \mathrm{ml})$ was about 2 -fold higher than the reported $\mathrm{C}_{\max }$ in normal mice treated with a similar dose but with a different formulation $(25 \mathrm{mg} / \mathrm{kg}$ tosylate salt administered in a simple aqueous suspension formulation, $C_{\max }=6.2 \mu \mathrm{g} / \mathrm{ml}$ at 1 hour) (16). However, at 24 hours, there was substantially more compound present after administration to the FRG huHep mice than for normal mice ( $8.5 \mathrm{vs} .0 .008 \mu \mathrm{g} /$ $\mathrm{ml})$, which may reflect differences in hepatic clearance between the humanized and nonhumanized mice. 


\section{A}

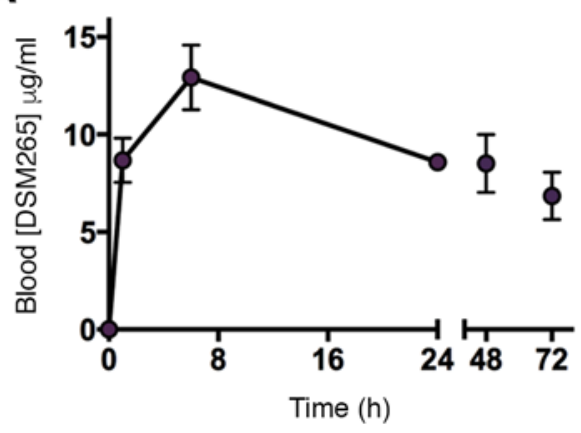

C

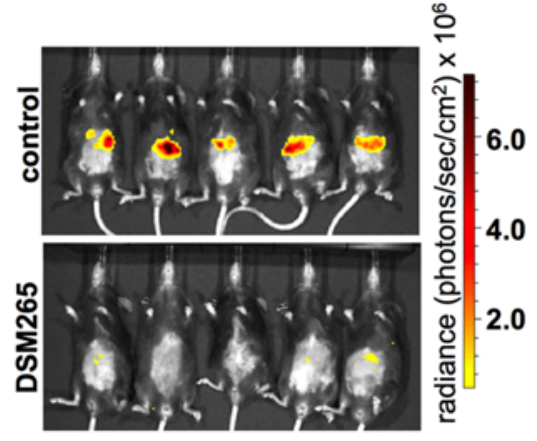

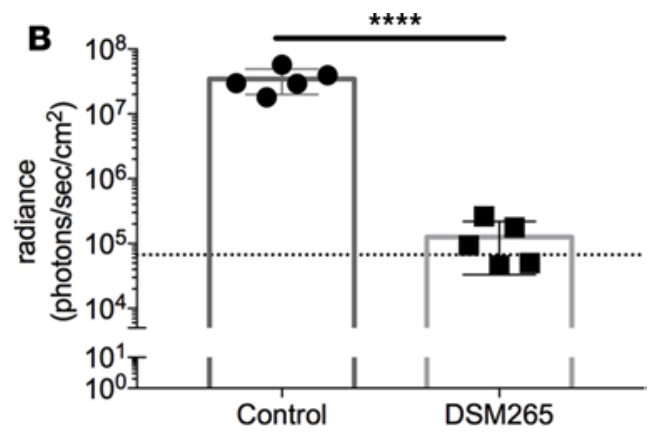

D

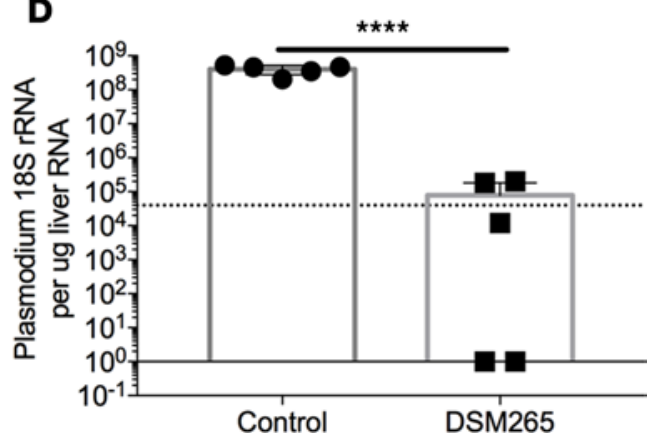

Figure 2. In vivo liver stage efficacy of DSM265. (A) Average blood concentration of DSM265 in 5 FRG huHep mice following oral administration at 20 mg/ $\mathrm{kg}$ daily for 3 days. (B) FRG huHep mice were infected by the probing of 50 infectious mosquitoes. DSM265 was administered $20 \mathrm{mg} / \mathrm{kg}$ free base 2 hours after infection and 24 and 48 hours after infection. Control mice were administered vehicle ( $5 \%$ methylcellulose) at the same time points. Liver burden was quantified on day 6 after infection by in vivo imaging. Dashed line denotes background radiance. (C) IVIS images of luminescence in livers of live FRC huHep mice from B. (D) P. falciparum liver burden measured by 18S rRNA qPCR. Dashed line denotes quantification limit of the assay. Unpaired 2-tailed Student's $t$ test, ${ }^{* * *} P<0.0001$. Each symbol represents 1 mouse (B and $\left.\mathbf{D}\right)$.

To determine if DSM265 showed efficacy against $P$. falciparum liver stages specifically, we treated mice with $20 \mathrm{mg} / \mathrm{kg}$ DSM265 at 2, 24, and 48 hours after infection. Liver parasite burden was quantified by IVIS and showed a $2.5-\log$ reduction in liver burden $(P<0.0001)$ in the DSM265-treated mice compared with control mice (Figure 2, B and C). Whole livers were also harvested on day 6 to determine parasite burden using qPCR. DSM265 resulted in a 5.2-log decrease in liver stage burden $(P<0.0001)$ (Figure 2D). Additionally, in 3 of the 5 mice treated with DSM265, the number of Plasmodium 18S rRNA copies were below the assay limit of quantification and were completely undetectable in 1 mouse.

Pharmacokinetic (PK) properties of primaquine $(P Q)$ in the FRG huHep model. To further exemplify the benefit of using the FRG huHep model for drug metabolism, we analyzed the metabolism of PQ, for which there is an abundance of clinical data available for comparison. PQ therapy affords causal prophylaxis against both $P$. falciparum and $P$. vivax (17) and is the only licensed drug that affords radical cure of $P$. vivax relapsing malaria $(17,18) . \mathrm{PQ}$ is metabolized in the liver, although the active metabolites remain unknown (19). Initially, to understand the breakdown of $\mathrm{PQ}$ to its primary metabolite, carboxyprimaquine (cPQ), and the effect of route of administration, FRG huHep mice were treated with $60 \mathrm{mg} / \mathrm{kg}$ PQ either by oral gavage or i.p. injection (Figure 3, A and B), and plasma was sampled at 3 minutes and 3, 12, and 24 hours after drug administration. The total plasma concentrations of $\mathrm{PQ}$ and of $\mathrm{CPQ}$ in the samples were quantified using Liquid chromatography-tandem mass spectrometry (LC-MS/MS) (20). Initially, PQ and cPQ concentrations were higher in the mice dosed by i.p. injection versus oral administration, reflecting potential differences in the rate of absorption, but terminal half-lives were comparable (Figure 3, A and B). Plasma levels of $\mathrm{PQ}$ and $\mathrm{CPQ}$ declined at similar rates over 24 hours, and overall exposure was higher for $\mathrm{cPQ}$ as previously described in animals (21).

To further understand the clearance of $\mathrm{PQ}$ in the liver-chimeric mouse compared with nonhumanized mice, we administered $60 \mathrm{mg} / \mathrm{kg}$ PQ orally to FRG huHep and Swiss Webster albino mice and measured blood PQ and $\mathrm{CPQ}$ levels over a 24-hour interval (Figure 3, C and D). Blood PQ and $\mathrm{cPQ}$ 

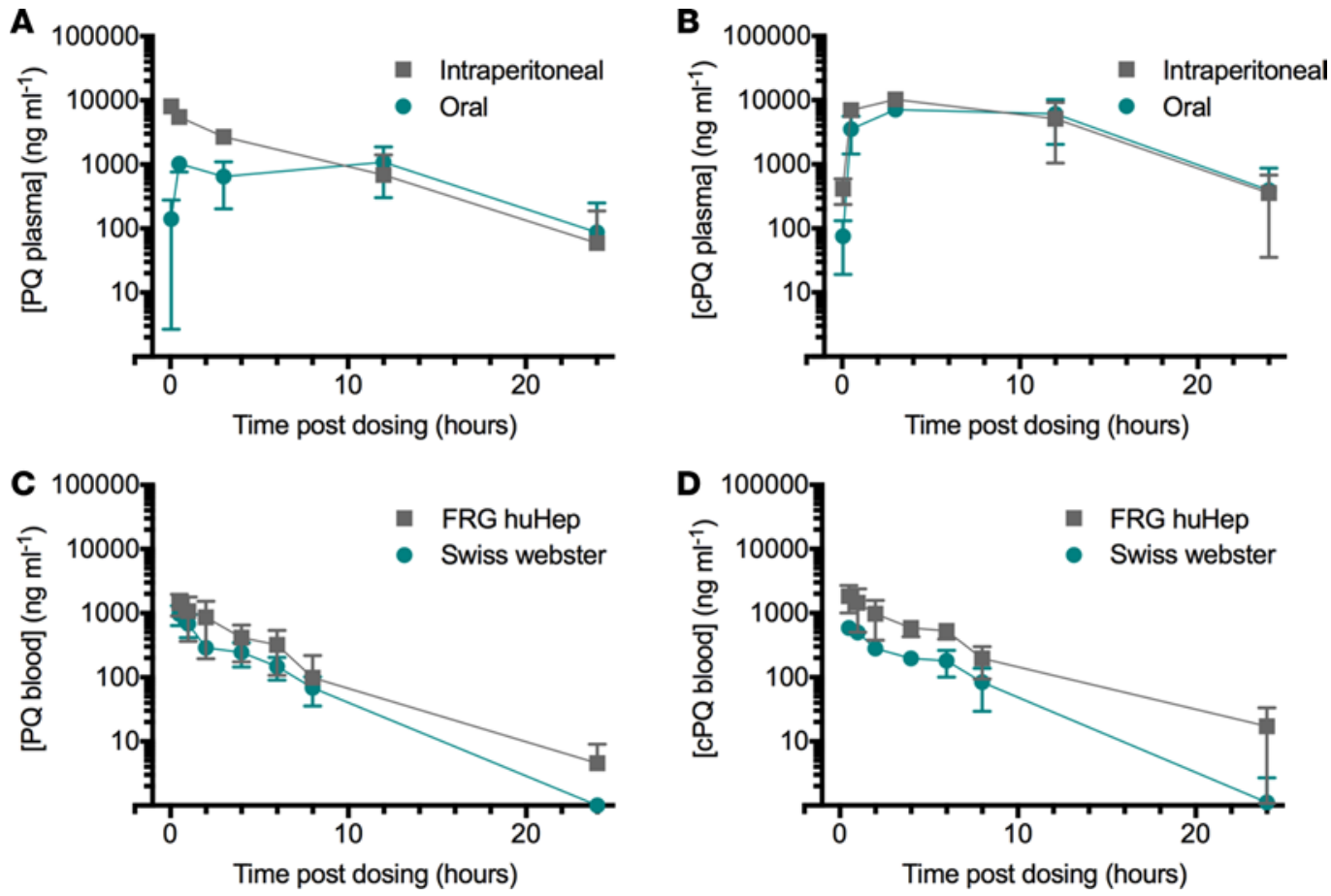

Figure 3. Concentrations of primaquine and carboxyprimaquine in FRG huHep mice. Comparison of average primaquine (PQ) (A) and carboxyprimaquine (CPQ) (B) plasma concentrations in FRG huHep mice administered $60 \mathrm{mg} / \mathrm{kg}$ primaquine either by i.p. injection (squares) or oral gavage (circles). Plasma samples were analyzed 3 minutes and 3, 12, and 24 hours after PQ administration in 3 mice, and concentrations were determined using LC-MS/MS. Comparison of average $\mathrm{PQ}(\mathbf{C})$ and $\mathrm{CPQ}$ (D) blood concentrations in FRG huHep (squares) or Swiss Webster (circles) mice administered $60 \mathrm{mg} / \mathrm{kg} \mathrm{PQ}$ orally. Whole blood was sampled $0.5,1,2,4,6,8$, and 24 hours after administration in 5 mice from each strain.

levels were consistently lower in Swiss Webster compared with FRG huHep mice (Table 1). While there were no significant differences for PK parameters of PQ between the 2 mouse strains, the AUC between 0 and 24 hours $\left(\mathrm{AUC}_{0-24}\right.$ ) and $\mathrm{C}_{\max }$ were higher for $\mathrm{CPQ}$ in the FRG huHep mice as compared with the Swiss Webster mice (Table 1). Furthermore, the ratio of $\mathrm{CPQ} / \mathrm{PQ}$ parameters were $>1$ in the FRG huHep mice and in published human data (22) compared with nonhumanized mice, where the ratio was $<1$. This suggests that $\mathrm{PQ}$ clearance in the liver-chimeric mice is lower than that in the nonhumized mice and possibly more similar to that in humans.

Single-dose PQ treatment significantly reduces $P$. falciparum liver stage burden. Having established that $P Q$ is metabolized in the liver-chimeric mice, we sought to determine the functional effect of PQ administered at different days during $P$. falciparum liver stage development. Previous studies in humans have shown that single-dose PQ efficacy for preventing blood stage infection is dependent on day of treatment (23). FRG huHep mice were infected by either the bite of 50 infected mosquitoes or i.v. injection of $1 \times 10^{6}$ salivary gland sporozoites. PQ $(60 \mathrm{mg} / \mathrm{kg})$ was administered orally 24 hours (early trophozoite stage) or 4 days (early schizogony stage) after infection. A single PQ dose 24 hours after infection afforded significant reduction in liver stage parasite burden whether inoculation was by mosquito bite infection (5.7-log reduction compared with vehicle-treated mice, $P<0.0001)$ or i.v. administration (4.5-log reduction, $P<0.01$ ) measured by qPCR (Figure 4). Plasmodium $18 \mathrm{~S}$ rRNA transcripts were undetectable in 2 of 3 mice infected by i.v. tail vein injection and 1 of 3 mice infected by mosquito bite. Although PQ administered on day 4 resulted in a 3.5- and 1.7-log reduction in liver stage parasite burden by mosquito bite infection or i.v. injection, respectively (measured by qPCR), the magnitude of effect was less than when FRG huHep mice were treated one day post-infection.

Liver stage-to-blood stage transition to determine viability of parasites in animals with reduced liver burden. To further assess the functional effect $\mathrm{PQ}$ had on parasites still present in the liver after treatment, FRG huHep mice were infused with human RBCs (hRBCs) to capture parasite egress. Engraftment of hRBCs began 5 days after infection and was steady over several days (Figure 5A). FRG huHep mice infected by mosquito bite were given $60 \mathrm{mg} / \mathrm{kg}$ PQ 1 or 4 days after infection, and the liver 
Table 1. Oral pharmacokinetic parameters of PQ and CPQ

\begin{tabular}{|c|c|c|c|}
\hline & Human $^{A}$ & FRG huHep & Swiss Webster \\
\hline Dose & $45 \mathrm{mg} P O$ & $60 \mathrm{mg} / \mathrm{kg} \mathrm{PO}$ & 60 mg/kg PO \\
\hline \multicolumn{4}{|l|}{$\mathbf{P Q}$} \\
\hline$C_{\max }(\mathrm{ng} / \mathrm{ml})$ & $65(34)$ & $1,434(475)$ & $971(334)$ \\
\hline $\mathrm{T}_{\max }(\mathrm{h})$ & $2(1)$ & $0.5(0)$ & $0.5(0)$ \\
\hline$t_{1 / 2}(h)$ & $4.4(1.4)$ & 7.2 (1.9) & $6.1(0.5)$ \\
\hline$A U C_{0-24}(n g / m l)$ & 468 (229) & $5,211(2,743)$ & 3658 (1585) \\
\hline$A \cup C_{0-\infty}(n g / m l)$ & & $5,269(2,730)$ & $3,667(1,585)$ \\
\hline \multicolumn{4}{|l|}{ CPQ } \\
\hline$C_{\max }(\mathrm{ng} / \mathrm{ml})$ & $736(236)$ & $1,844(843)$ & $541(63)^{B}$ \\
\hline $\mathrm{T}_{\max }(\mathrm{h})$ & $8(2)$ & $0.5(0)$ & $0.5(0)$ \\
\hline$t_{1 / 2}(h)$ & & $5.7(0.4)$ & $6.6(0.6)^{\mathrm{B}}$ \\
\hline$A U C_{0-24}(\mathrm{ng} / \mathrm{ml})$ & $1,420(530)$ & $7,620(2,769)$ & $2,669(621)^{\mathrm{B}}$ \\
\hline$A U C_{0-\infty}(n g / m l)$ & & $7,759(2,734)$ & $2,687(630)^{\mathrm{B}}$ \\
\hline \multicolumn{4}{|l|}{$\mathrm{cPQ} / \mathrm{PQ}$ ratios } \\
\hline$C_{\max } c P Q / P Q$ & 11.3 & 1.3 & 0.6 \\
\hline $\mathrm{AUC}_{0-24} \mathrm{cPQ} / \mathrm{PQ}$ & 3.0 & 1.5 & 0.7 \\
\hline \multicolumn{4}{|c|}{$\begin{array}{l}\text { APlasma concentration data from ref. } 22 \text {. Five each of FRG huHep and Swiss } \\
\text { Webster mice were used for pharmacokinetic estimates. }{ }^{B} P<0.05 \text {; significantly } \\
\text { different from FRG huHep; } 2 \text {-tailed Student's } t \text { test. Averages are reported with SD } \\
\text { in parenthesis. h, hour; } C_{\text {max' }} \text {, maximum drug concentration; } T_{\text {max }} \text {, time at maximum } \\
\text { drug concentration; } \mathrm{t}_{1 / 2} \text {, half-life; } A U C \text {, area under the curve; } P Q \text {, primaquine; } C P Q \text {, } \\
\text { carboxyprimaquine; } P O \text {, by mouth. }\end{array}$} \\
\hline
\end{tabular}

stage burden was measured by IVIS (Figure 5B). Peak IVIS signal was observed 6 days after infection, and this signal dropped dramatically as parasites egressed out of the liver and into the blood stream on day 7. Although the luciferase expression in the parasite is constitutive, early blood stage infection is undetectable due to a combination of the dilution of the parasites in the mouse periphery and the lower levels of luciferase expression in the ring stage of the parasite compared with that of liver schizonts. Mice treated with PQ on day 1 had no detectable IVIS signal throughout liver stage development; however, in 1 of 3 mice, $18 \mathrm{~S}$ rRNA transcript was detected 9 days after infection (Figure 5C). This is a 2-day delay in onset of patency compared with the untreated mice, where 5 of 5 animals became patent 7 days after infection. Mice treated with PQ 4 days after infection showed sustained levels of liver parasite burden with no sudden increase or decrease in IVIS signal. Animals became blood stage patent in this group on days 7, 8 and 9 , yet 1 animal did not develop blood stage infection by day 9. Liver stage-to-blood stage transition was assessed in FRG huHep mice treated with ATQ and PG (30 and 10 $\mathrm{mg} / \mathrm{kg}$, respectively) 1 day prior to mosquito bite infection and each subsequent day for 6 days. In the treatment group, $18 \mathrm{~S}$ rRNA transcripts were not detectable in any mice 7 and 8 days after infection. Nine days after infection, 2 of the 5 mice exhibited very low levels of $18 \mathrm{~S}$ rRNA, below the quantitation limit of the assay (Figure 5D).

\section{Discussion}

The discovery of potentially novel antimalarial compounds continues to be an important facet of the global effort to eradicate malaria. Historically, antimalarial drug discovery has focused on the symptomatic blood stages of the infection. The importance of identifying multistage active compounds - those that can prevent liver infection and transmission, as well as cure symptomatic blood stage infections - is becoming increasingly appreciated (8). The challenge of developing liver stage active compounds is highlighted by the recently developed spiroindolone class of antimalarial drugs, which - despite having remarkably potent blood stage killing kinetics (median parasite clearance half-life of 0.9 hours) and a potentially novel target (PfATP4) - have failed to demonstrate significant liver stage activity $(24,25)$. One major barrier in the development of liver stage active compounds is the lack of a model system that sufficiently reproduces the complexity of human liver infection with $P$. falciparum. This directly limits our ability to preclinically assess liver stage activity and therefore predict outcomes in humans. Here, we use the liver-chimeric FRG huHep mouse model to test the efficacy of antimalarial compounds against $P$. falciparum liver stages in vivo.

The FRG huHep model is well suited for testing late-lead and preclinical drug candidates to identify their inhibitory effect on $P$. falciparum liver stage development prior to testing in humans. Here, we validate the model using therapies well characterized in humans. The first therapy, ATQ-PG (also referred to as Malarone) combination therapy, is a standard prophylactic treatment that prevents liver stage infection in humans (26). Using the same daily dosing schedule recommended for travelers, ATQ-PG treatment reduced liver burden to undetectable levels and prevented liver stage-to-blood stage transition in 3 of 5 mice. The qPCR assay used is capable of detecting 20 P. falciparum ring-stage parasites per ml of blood equivalent to about $7 \times 10^{4}$ Plasmodium $18 \mathrm{~S} \mathrm{rRNA}$ copies $/ \mathrm{ml}$ of blood. In the other 2 mice, $18 \mathrm{~S}$ rRNA levels were well below the quantitation limit.

Additionally, we used PQ to confirm drug metabolism in the model and parasite killing kinetics. Arnold et al. demonstrated the importance of the timing of action of PQ treatment in volunteers administered $P$. falciparum by mosquito bite. In that study, single-dose treatment with PQ 1 day after mosquito bite protected $100 \%$ of volunteers from blood stage infection (27). Treatment on day 3 or 5 after infection 

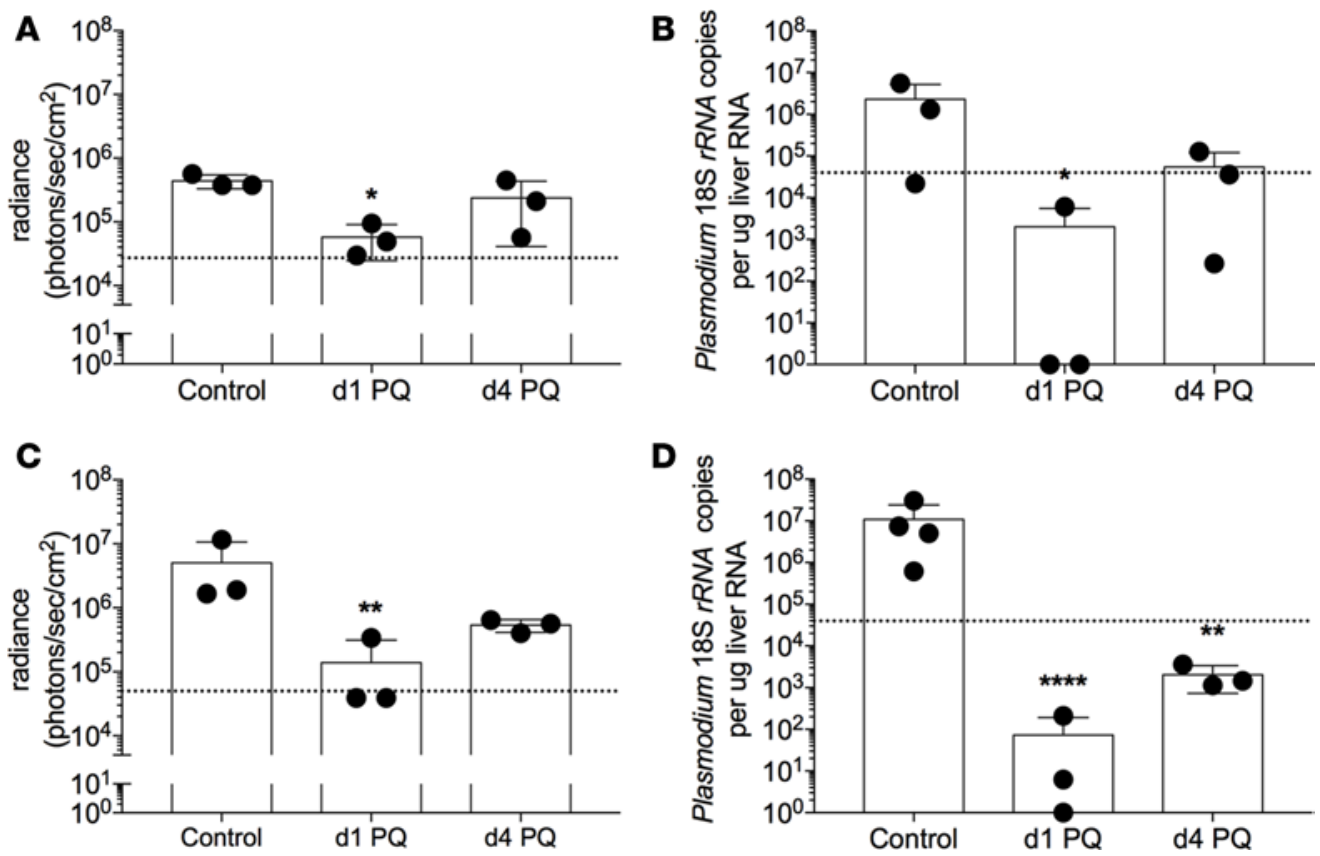

Figure 4. In vivo $\boldsymbol{P}$. falciparum liver stage burden in FRG huHep mice following primaquine treatment. $P$. falciparum liver burden measured by (A) in vivo imaging and (B) 185 rRNA qPCR following infection with $1 \times 10^{6}$ sporozoites by tail vein injection. Two groups of mice were treated 1 or 4 days after infection with single-dose $60 \mathrm{mg} / \mathrm{kg}$ primaquine (PQ) orally. $P$. falciparum liver burden was measured by (C)in vivo imaging or (D) 185 rRNA qPCR following infection by the bite of 50 infected mosquitoes. Two groups were treated with $\mathrm{PQ}$ as described above. Each symbol represents 1 mouse. Dashed lines in $\mathbf{A}$ and $\mathbf{C}$ denote background radiance. Dashed lines in $\mathbf{B}$ and $\mathbf{D}$ denote quantification limit of the assay. One-way ANOVA with Dunnett's correction for multiple testing, ${ }^{*} P<0.05,{ }^{* *} P<0.01,{ }^{* * *} P<0.0001$.

resulted in protection of $90 \%$ and $10 \%$ of volunteers, respectively, highlighting the importance of timing of therapy. Our results mimic those observed in humans, as we see a greater reduction in liver burden when PQ is given 1 day after infection compared with 4 days after infection and reductions in parasite transition to blood stage in animals treated 1 day after infection compared with 4 days after infection. Although we did detect 18S rRNA transcripts in the "1-day after infection" treatment group, these levels were well below the quantitative limit of the assay, and this residual rRNA may not represent patent bloodstream infection. Furthermore, the failure to achieve complete protection ( 2 of 3 mice were protected) could be due to the number of sporozoites inoculated: 1 million in our experiment by i.v. injection or 50 mosquito bites compared with 10 mosquito bites in ref. 27. Importantly, the FRG huHep system also allowed us to analyze PQ PKs, where appearance of $\mathrm{cPQ}$ is commonly used as a proxy for PQ metabolism (28). The ratios of $\mathrm{cPQ} /$ PQ were more similar to humans in the liver-chimeric mice than normal mice, suggesting the FRG huHep mice more closely resemble the PK observed in humans.

To further exemplify the potential of the FRG huHep platform, we tested the effect of a drug candidate, DSM265, against in vivo $P$. falciparum liver stages. DSM265 has potent blood stage $P$. falciparum activity against the replicating parasite (half-maximal effective concentration $\left[\mathrm{EC}_{5}\right]=0.0046 \mu \mathrm{g} / \mathrm{ml}$ ) $(16)$ and reduces the size of liver stages in vitro $\left(\mathrm{EC}_{50}=0.0057 \mu \mathrm{g} / \mathrm{ml}\right)$. Therefore, we were interested in testing its ability to prevent liver stage development in vivo. Treatment with DSM265 led to a substantial reduction in parasitic liver burden when assessed by qPCR. The minimum blood levels achieved in this study with daily dosing were about 8-fold above the reported level required for a minimum parasiticidal clearance in the blood stage SCID model (16). It is important to note that a full assessment of the $\mathrm{PK} /$ pharmacodynamic relationships for DSM265 in this liver stage model was not a goal of this proof-of-concept study, and thus, there is insufficient data to make conclusions about the relative efficacy of DSM265 on liver stage versus blood stage. DSM265 was recently tested in 2 separate phase I clinical studies $(29,30)$, including one that evaluated prophylactic potential. In this study, it showed complete protection when a single dose was administered 1 day prior to $P$. falciparum infection, while it only provided partial protection when administered 7 days prior to infection, again highlighting the importance of timing of action and PKs. In this preclinical study, the peak plasma concentration after a single $400 \mathrm{mg}$ dose was $11.5 \mu \mathrm{g} / \mathrm{ml}$, a concentration lower than the peak blood concen- 
A

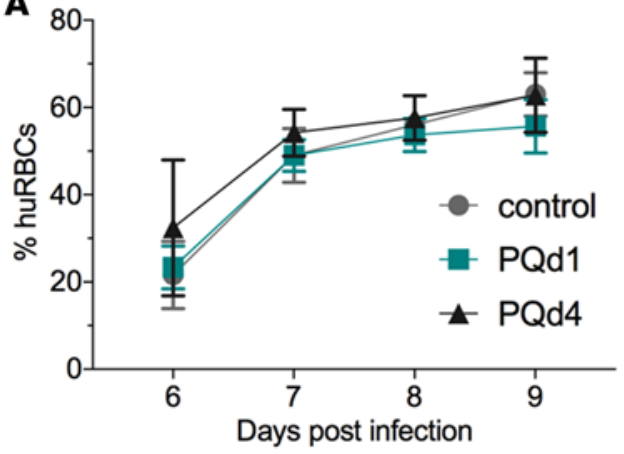

C

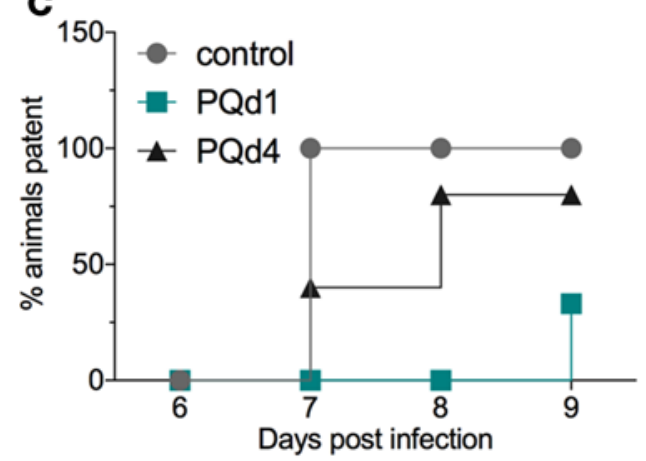

B

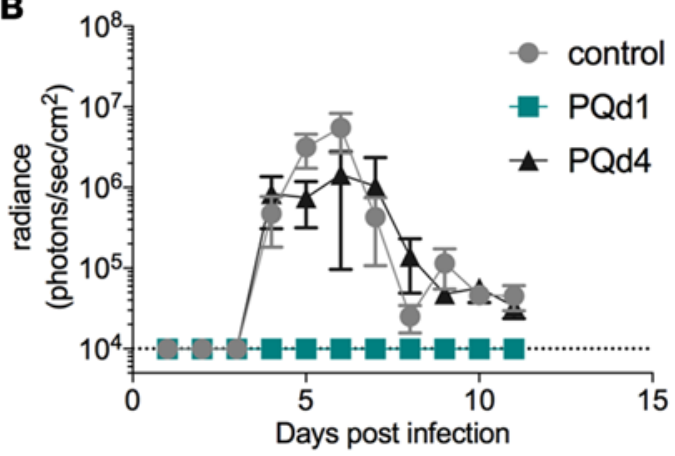

D

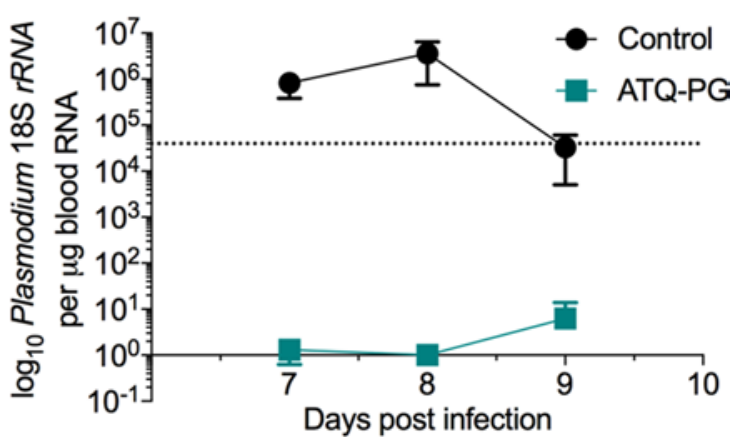

Figure 5. Liver stage-to-blood stage transition of $\boldsymbol{P}$. falciparum in FRG huHep mice. (A) Average percent human RBC (hRBC) engraftment in FRG huHep mice (3 each group) following hRBC injections beginning 5 days after infection measured by flow cytometry. (B) Time course of liver stage burden quantified by in vivo imaging days 1-11 after infection. Dashed line denotes background radiance counts. Three mice were measured in each group. (C) Survival curve demonstrating the transition of $P$. falciparum parasites from the liver to the blood. Blood stage patency was determined by Plasmodium 185 rRNA qPCR 6-9 days after sporozoite infection. Mice were considered blood stage-positive if any number of transcripts was detectable. Three mice per group. (D) Plasmodium 18S rRNA levels in the blood following mosquito bite infection and treatment with atovaquone (30 mg/kg) and proguanil (10 mg/kg) from 1 day prior to mosquito bite to 6 days after infection. Five mice per group.

tration observed in the FRG huHep mice after a single $20 \mathrm{mg} / \mathrm{kg}$ dose. DSM265 exhibits a half-life between 86 and 118 hours in humans, further highlighting its potential as an advanced causal prophylactic therapy.

In our assessment of the FRG huHep model, we found great utility of the model due to the versatility of the route of sporozoite infection. We demonstrate that robust liver stage infection can be achieved by the bite of P. falciparum-infected mosquitoes - an important finding, as it mimics the natural route of infection in humans. Infection by mosquito bite or i.v. injection lends significant adaptability to the system, potentially allowing for the use of i.v.-administered cryopreserved sporozoites (31) in laboratory settings where there is no access to $P$. falciparum-infected mosquitoes. While there was no statistically significant difference in parasite liver burden between mosquito bite and i.v. injection (whether measured by IVIS or qPCR), the similar or slightly elevated liver burden in the mosquito-bitten mice is potentially surprising considering that only 10-100 sporozoites are expected to be injected per mosquito (6). Nevertheless, the fitness as well as infectivity of sporozoites dissected from the salivary glands most likely varies when compared with sporozoites ejected from the salivary glands during mosquito bite - the physiological route of Plasmodium sporozoite infection.

Liver infection was assessed using an IVIS system or qPCR with similar results. While the dynamic range is greater in the qPCR assay, the ability to use IVIS technology in the model system is vital, as it allows for the execution of time course experiments without the need to sacrifice animals or harvest tissue (Figure 6). We were additionally able to show that, while liver burden was reduced when PQ was given 4 days after infection, the levels of liver infection were stable over time, suggesting either that parasites are arrested and continue to persist, yet cannot replicate, or that a majority of the parasite load is killed by the treatment and only a few continue to grow, albeit at a less synchronous course. This observation is further supported by the delay in onset of patency observed in the mice treated 4 days after infection. This feature could be important for future studies assessing the killing kinetics of candidate drugs. Importantly, because 1 liver stage parasite can initiate blood stage infection, our liver stage model system will make it possible to quantify liver stage infection with 1 
generation of mature $P$. falciparum NF54HT-GFP-luc gametocytes

(16 days)

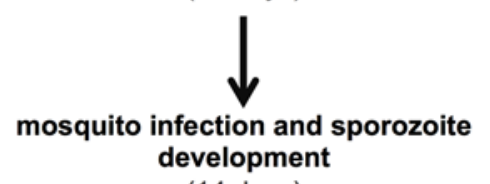

(14 days)
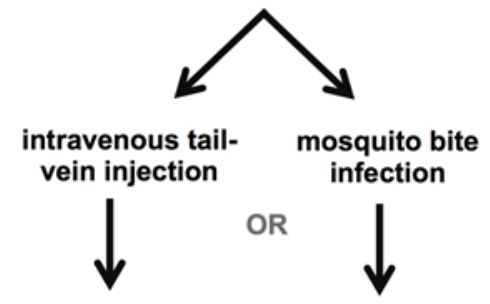

liver-stage development (0-7 days)

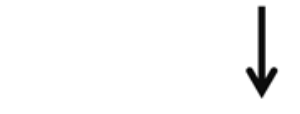

measurement of liver-stage burden ( $>4$ days post-infection)

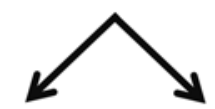

in vivo imaging $\mathrm{OR} \quad$ qRT-PCR

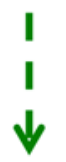

Liver to blood-stage

transition

(7 days post

infection)
Figure 6. Experimental design for testing liver stage efficacy of antimalarials against $\boldsymbol{P}$. falciparum. Mature, luciferase-expressing $P$. falciparum (Pf NF54 GFP-luc) gametocytes are produced in vitro in hRBC culture. Mosquitoes are infected with mature stage $V$ gametocytes using a standard membrane feeding assay. Fourteen days after feeding, FRG huHep mice are infected by mosquito bite or i.v. injection with freshly dissected salivary gland sporozoites. $P$. falciparum liver parasite burden is quantified 4-6 days after infection by live in vivo bioluminescence imaging or liver harvest and Plasmodium 185 rRNA qPCR. Optionally, injection of hRBCs on day 5 after infection and subsequent days captures the liver stage-to-blood stage transition on days 7-9. drug administration parasite by measuring the liver stage-to-blood stage transition, the most sensitive detection method of prophylactic liver stage drug efficacy. Current methods to test liver stage efficacy of preclinical compounds utilize an in vitro $P$. falciparum model to test the effect on invasion and growth (32) or rodent malaria models for testing in vivo causal prophylaxis (33). While these systems are attractive due to scalability and cost effectiveness, they are limited in regards to the applicability of in vitro results and generalizability between rodent and human malaria species. Liver stage development in humans is 4 days longer than that of rodent malaria parasite species; therefore, differences in life cycle processes may affect drug efficacy in the 2 different models. While the cost is much higher for the FRG huHep mice than normal mice, the FRG huHep mice and nonhuman primates are the only in vivo animal models available to test drug efficacy against $P$. falciparum liver stages. The FRG huHep model represents great cost savings over the nonhuman primate models and produces more robust and complete data, as several drug concentrations can be used and repeated in several animals. The FRG huHep system could, thus, allow promising lead compounds to be more accurately tested against liver stage $P$. falciparum and improve prioritization for clinical trial consideration. In doing so, the P. falciparum FRG huHep infection model in liver-chimeric mice fills a gap in the antimalarial drug development pipeline. It will be of interest to test in the future whether the FRG huHep system is better at predicting clinical outcomes of drug treatments than other currently existing preclinical models.

\section{Methods}

\section{P. falciparum sporozoite production and mouse infection}

The $P$. falciparum NF54HT-GFP-luc parasite, which constitutively expresses luciferase under the EF1a promoter (15), was maintained in RPMI 1640 (25 mM HEPES, 2 mM L-glutamine) supplemented with 50 $\mu \mathrm{M}$ hypoxanthine plus $10 \%$ human serum and subcultured in $5 \% \mathrm{O}^{+}$human erythrocytes (Interstate Blood Bank). Mature sexual-stage gametocytes were induced as previously described (11). Briefly, asexual cultures were inoculated at $1 \%$ parasitemia with no further subculturing and daily media changes to induce gametocytogenesis. Mature gametocytes (day 16 after induction) were fed to 4-day-old Anopheles stephensi mosquitoes to initiate infection. Mosquitoes were incubated at $27^{\circ} \mathrm{C}$ and $75 \%$ humidity for 14 days and given $8 \%$ dextrose + para-aminobenzoic acid (PABA) to foster parasite growth. FRG-KO on C57B1/6 human repopulated mice were purchased from Yecuris Corporation and exhibited primary human hepatocyte repopulation levels above $70 \%$, as determined by human serum albumin levels. All mice within the same experiment were engrafted with primary human hepatocytes originating from the same donor selected from an assortment of prequalified lots. Mice were cycled on NTBC once a month for 4 days at $8 \mathrm{mg} / 1$ in drinking water to maintain health. Animals did not receive NTBC 3 weeks prior to and during drug studies. For i.v. injection into the retroorbital plexus or tail vein, 14- to 19-day-old mosquito salivary glands were dissected, purified, and resuspended in a volume of 1 million sporozoites per $100 \mu 1$ in Schneider's medium. For mosquito bite infection, 3-5 FRG huHep mice were anesthetized and placed on the top of 
a mosquito cage containing 150-250 infected mosquitoes for 10 minutes; therefore, each mouse received probing by approximately 50 mosquitoes. Mice were repositioned every 120 seconds to encourage probing by the mosquitoes (34).

\section{Quantification of liver stage parasite burden}

Whole animal live bioluminescence imaging. Luciferase activity was measured in the FRG huHep mice using the IVIS Lumina II animal imager (Perkin Elmer). The abdomen of FRG huHep mice were shaved for unobstructed signal detection. Mice were injected i.p. with $100 \mu 1$ XenoLight RediJect D-Luciferin (PerkinElmer) to quantitate bioluminescence. Animals were anesthetized and imaged within 5 minutes of luciferin injection. Signal was acquired for 5 minutes using a field of view of $10 \mathrm{~cm}$ and medium binning factor. Living Image 3.0 software was used to measure total flux (photons/second) of a region of interest (ROI), which was placed around the location of the liver in each mouse. A second ROI was placed elsewhere on the mouse to determine background signal. Luminescence signal is generally visible between 4 and 6 days after the sporozoite infection.

$q P C R$. FRG huHep mice were euthanized on day 6 of infection, and livers were harvested for qPCR quantification. Each liver lobe was cut in half and added to TRIzol reagent (Invitrogen), pooling all lobes for each mouse. Livers were homogenized and RNA extraction was performed using the RNeasy purification kit (Qiagen). Quantification of parasite liver burden by 18S rRNA qPCR (or detection of the parasite in $\mathrm{hRBCs}$ ) was done as previously described (35) with modifications as follows due to the liver tissue presence: pan-Plasmodium 18S rRNA primers and a probe were as reported (36), and results from the extracted, in vitro-transcribed RNA standard curve were normalized to a duplexed reaction for human GAPDH mRNA to control for percentage of human hepatocyte repopulation. Human GAPDH mRNA-specific reagents were the PrimeTime Predesigned qPCR Assay (HsPT.39a.22214836; IDT).

\section{P. falciparum liver stage-to-blood stage transition}

Five days after infection, FRG huHep mice were injected in the retroorbital plexus with $50 \mu 1$ clodronate-containing liposomes (Clophosome-A, FormuMax), to clear macrophages, and $500 \mu \mathrm{hRBCs}(70 \%$ $\mathrm{O}^{+}$human erythrocytes in RPMI 1640 (25mM HEPES, 2mM L-glutamine) supplemented with $50 \mu \mathrm{M}$ hypoxanthine plus $10 \%$ human serum). The next day, mice were bled $200 \mu 1$ and received an i.p. injection of $700 \mu \mathrm{hRBCs}$. Seven days after challenge, the percentage of $\mathrm{hRBCs}$ reached $50 \%$ (measured as described before by flow cytometry; ref. 37). From day 6-9, each mouse was bled $100 \mu \mathrm{l}$ from the retroorbital plexus and injected i.p. with $500 \mu \mathrm{hRBCs}$ to keep the percentage of hRBCs stable between $50 \%$ and $70 \%$. If the percentage reached more than $70 \%$, the mice were not injected with hRBCs. Blood samples from days 6-9 were stored in TRIzol, and quantification of parasite burden by qPCR specific to Plasmodium 18S rRNA was done as described above. Additional blood samples were not taken to minimize the number of bleeds performed on these animals.

\section{Compounds}

PQ was purchased from MilliporeSigma (catalog 160393), reconstituted in PBS, and filter sterilized for administration to animals. A $60 \mathrm{mg} / \mathrm{kg}$ dose was chosen to ensure activity in the humanized mice. DSM265 was prepared as an amorphous spray dried dispersion (SDD) containing 25\% DSM265 (16). Solid material was formulated in $0.5 \%$ methylcellulose and dosed by oral gavage as a suspension at $80 \mathrm{mg} / \mathrm{kg}$ of the SDD formulation, resulting in a dose of $20 \mathrm{mg} / \mathrm{kg}$ DSM265 free base. ATQ and PG were purchased from MilliporeSigma (catalogs A7986 and G7048, respectively) and reconstituted in $0.5 \%$ methycellulose $/ 0.5 \%$ tween. The compound was given daily to follow the same dosing schedule followed in humans. The concentration of dose was chosen at $30 \mathrm{mg} / \mathrm{kg}$ and $10 \mathrm{mg} / \mathrm{kg}$ for ATQ and PG, respectively. The ATQ dose was chosen as the dose that is within the range of the dose efficacious in the literature, and PG was chosen as a dose about 3-fold lower as is used in the treatment of humans.

\section{Compound concentrations in FRG huHep plasma}

For the determination of $\mathrm{PQ}$ and $\mathrm{cPQ}$ concentrations, 3 mice each were administered $60 \mathrm{mg} / \mathrm{kg} \mathrm{PQ}$ either by oral gavage or i.p. injection. To compare the route of administration, blood samples of $100 \mu 1$ were collected into tubes containing anticoagulant at 3 minutes and 3, 12, and 24 hours after administration. Plasma was separated by centrifugation and prepared by solid-phase extraction and eluate concentrations 
determined using LC-MS (20). To compare FRG huHep with Swiss Webster mice, 10- $\mu$ l volumes of blood were collected using Mitra Microsampling Devices (Neoteryx). Determination of the DSM265 concentrations in FRG huHep mice was conducted following 3 daily doses of $20 \mathrm{mg} / \mathrm{kg}$ free base. Whole blood was collected 3 hours after the first dose and then just prior to the next dose 24, 48, and 72 hours later. Blood samples were collected using Mitra Microsampling Devices as above. Samples were then extracted with methanol $(100 \mu 1)$, followed by vortex mixing and brief sonication, and an aliquot of the supernate was analyzed by LC-MS. Concentrations of DSM265 were quantitated by comparison to a calibration curve prepared using control blank mouse blood. All concentrations represent total blood concentrations and are not adjusted for binding to plasma proteins.

\section{Statistics}

Pearson correlation, unpaired 2-tailed Student's $t$ test and 1-way ANOVA with Dunnett's correction for multiple testing were used and were conducted using GraphPad Prism 6.0 for Mac. Error bars represent mean \pm SD unless otherwise noted. $P$ values less than 0.05 were considered significant. When Student's $t$ tests were used for analyses, they were 2-tailed.

\section{Study approval}

All animal procedures were conducted in accordance with and approved by the Center for Infectious Disease Research IACUC under protocol SK-16. The Center for Infectious Disease Research IACUC adheres to the NIH Office of Laboratory Animal Welfare standards (Office of Laboratory Animal Welfare [OLAW] welfare assurance A3640-01).

\section{Author contributions}

ELF, LF, CS, BKS, and SAM designed, conducted, and analyzed data for FRG huHep-infection experiments. VC, MF, MJN, WB, NC, and TN conducted FRG huHep-infection experiments. ZB, TMO, JL, and SCM conducted and analyzed qPCR experiments. ELF conducted and analyzed PQ PK studies. EMW and JB provided animals. BC and SHIK assisted in experimental design. SAC, JS, and MAP designed and analyzed data for experiments involving DSM265. ELF, SHIK, and SAM wrote the manuscript.

\section{Acknowledgments}

We would like to thank Omar Vandal, Richard Elliott, and Jeremy Burrows for helpful discussions concerning research presented in this manuscript. This work was funded by the Center for Infectious Disease internal funding sources; the United States NIH grant R01AI103947 (to MAP) and F32-AI102567-04 (to ELF); Medicines for Malaria Venture (to SAC); OPP1137694 from Bill and Melinda Gates Foundation (to SAM, SCM); Belgian American Educational Foundation (to LF); and the German Research Foundation, DFG (research grant SCHA 2047 /1-1; to CS). We would like to thank the insectary core at the Center for Infectious Disease Research for rearing of $A$. stephensi mosquitoes.

Address correspondence to: Sebastian A. Mikolajczak or Stefan H.I. Kappe, 307 Westlake Avenue N, Suite 500, Seattle, Washington 98109, USA. Phone: 206.256 .7200 (S.A. Mikolajczak); 206.256.7205 (S.H.I. Kappe).Email: sebastian.mikolajczak@cidresearch.org (S.A. Mikolajczak); stefan.kappe@cidresearch.org (S.H.I. Kappe).

1. Bhatt S, et al. The effect of malaria control on Plasmodium falciparum in Africa between 2000 and 2015. Nature. 2015;526(7572):207-211.

2. Miotto O, et al. Multiple populations of artemisinin-resistant Plasmodium falciparum in Cambodia. Nat Genet. 2013;45(6):648-655.

3. Roberts L. Skirmishing over the scope of the threat. Science. 2016;352(6284):403.

4. Burrows JN, van Huijsduijnen RH, Möhrle JJ, Oeuvray C, Wells TN. Designing the next generation of medicines for malaria control and eradication. Malar J. 2013;12:187.

5. malERA Consultative Group on Drugs. A research agenda for malaria eradication: drugs. PLoS Med. 2011;8(1):e1000402.

6. Vanderberg JP, Frevert U. Intravital microscopy demonstrating antibody-mediated immobilisation of Plasmodium berghei sporozoites injected into skin by mosquitoes. Int J Parasitol. 2004;34(9):991-996.

7. Kappe SH, Vaughan AM, Boddey JA, Cowman AF. That was then but this is now: malaria research in the time of an eradication agenda. Science. 2010;328(5980):862-866.

8. Diagana TT. Supporting malaria elimination with 21st century antimalarial agent drug discovery. Drug Discov Today. 2015;20(10):1265-1270. 
9. Hermsen CC, et al. Detection of Plasmodium falciparum malaria parasites in vivo by real-time quantitative PCR. Mol Biochem Parasitol. 2001;118(2):247-251.

10. Gujjar R, et al. Lead optimization of aryl and aralkyl amine-based triazolopyrimidine inhibitors of Plasmodium falciparum dihydroorotate dehydrogenase with antimalarial activity in mice. J Med Chem. 2011;54(11):3935-3949.

11. Vaughan AM, et al. Complete Plasmodium falciparum liver-stage development in liver-chimeric mice. J Clin Invest. 2012;122(10):3618-3628.

12. Azuma H, et al. Robust expansion of human hepatocytes in Fah-/-/Rag2-/-/Il2rg-/- mice. Nat Biotechnol. 2007;25(8):903-910.

13. Bissig KD, Le TT, Woods NB, Verma IM. Repopulation of adult and neonatal mice with human hepatocytes: a chimeric animal model. Proc Natl Acad Sci USA. 2007;104(51):20507-20511.

14. Mikolajczak SA, et al. Plasmodium vivax liver stage development and hypnozoite persistence in human liver-chimeric mice. Cell Host Microbe. 2015;17(4):526-535.

15. Vaughan AM, et al. A transgenic Plasmodium falciparum NF54 strain that expresses GFP-luciferase throughout the parasite life cycle. Mol Biochem Parasitol. 2012;186(2):143-147.

16. Phillips MA, et al. A long-duration dihydroorotate dehydrogenase inhibitor (DSM265) for prevention and treatment of malaria. Sci Transl Med. 2015;7(296):296ra111.

17. Kolifarhood G, et al. Prophylactic efficacy of primaquine for preventing Plasmodium falciparum and Plasmodium vivax parasitaemia in travelers: A meta-analysis and systematic review. Travel Med Infect Dis. 2017;17:5-18.

18. Schmidt LH, Fradkin R, Genther CS, Hughes HB. Delineation of the Potentials of Primaquine as a radical curative and prophylactic drug. Am J Trop Med Hyg. 1982;31(3):666-680.

19. Vale N, Moreira R, Gomes P. Primaquine revisited six decades after its discovery. Eur J Med Chem. 2009;44(3):937-953.

20. Page-Sharp M, Ilett KF, Betuela I, Davis TM, Batty KT. Simultaneous determination of primaquine and carboxyprimaquine in plasma using solid phase extraction and LC-MS assay. J Chromatogr B Analyt Technol Biomed Life Sci. 2012;902:142-146.

21. Mihaly GW, Ward SA, Edwards G, Nicholl DD, Orme ML, Breckenridge AM. Pharmacokinetics of primaquine in man. I. Studies of the absolute bioavailability and effects of dose size. Br J Clin Pharmacol. 1985;19(6):745-750.

22. Ward SA, et al. Pharmacokinetics of primaquine in man. II. Comparison of acute vs chronic dosage in Thai subjects. Br J Clin Pharmacol. 1985;19(6):751-755.

23. Arnold J, et al. The antimalarial action of primaquine against the blood and tissue stages of falciparum malaria (Panama, P-F-6 strain). J Lab Clin Med. 1955;46(3):391-397.

24. Rottmann M, et al. Spiroindolones, a potent compound class for the treatment of malaria. Science. 2010;329(5996):1175-1180

25. White NJ, et al. Spiroindolone KAE609 for falciparum and vivax malaria. N Engl J Med. 2014;371(5):403-410.

26. Berman JD, et al. Causal prophylactic efficacy of atovaquone-proguanil (Malarone) in a human challenge model. Trans $R$ Soc Trop Med Hyg. 2001;95(4):429-432.

27. Arnold J, et al. The antimalarial action of primaquine against the blood and tissue stages of falciparum malaria (Panama, P-F-6 strain). J Lab Clin Med. 1955;46(3):391-397.

28. Avula B, et al. Analysis of primaquine and its metabolite carboxyprimaquine in biological samples: enantiomeric separation, method validation and quantification. Biomed Chromatogr. 2011;25(9):1010-1017.

29. McCarthy JS, et al. Safety, tolerability, pharmacokinetics, and activity of the novel long-acting antimalarial DSM265: a two-part first-in-human phase 1a/1b randomised study. Lancet Infect Dis. 2017;17(6):626-635.

30. Sulyok M, et al. DSM265 for Plasmodium falciparum chemoprophylaxis: a randomised, double blinded, phase 1 trial with controlled human malaria infection. Lancet Infect Dis. 2017;17(6):636-644.

31. Kennedy M, et al. A rapid and scalable density gradient purification method for Plasmodium sporozoites. Malar J. 2012;11:421

32. March S, et al. A microscale human liver platform that supports the hepatic stages of Plasmodium falciparum and vivax. Cell Host Microbe. 2013;14(1):104-115.

33. Most H, Montuori WA. Rodent systems (Plasmodium berghei-Anopheles Stephensi) for screening compounds for potential causal prophylaxis. Am J Trop Med Hyg. 1975;24(2):179-182.

34. Sack BK et al. Humoral protection against mosquito bite-transmitted Plasmodium falciparum infection in humanized mice. NPJ Vaccines. 2017;2:27.

35. Murphy SC, Daza G, Chang M, Coombs R. Laser cutting eliminates nucleic acid cross-contamination in dried-blood-spot processing. J Clin Microbiol. 2012;50(12):4128-4130.

36. Billman ZP, Seilie AM, Murphy SC. Purification of Plasmodium Sporozoites Enhances Parasite-Specific CD8+ T Cell Responses. Infect Immun. 2016;84(8):2233-2242.

37. Angulo-Barturen I, et al. A murine model of falciparum-malaria by in vivo selection of competent strains in non-myelodepleted mice engrafted with human erythrocytes. PLoS One. 2008;3(5):e2252. 